\title{
Role of callose synthases in transfer cell wall development in tocopherol deficient Arabidopsis mutants
}

\author{
Hiroshi Maeda ${ }^{1,2,3 t}$, Wan Song ${ }^{1,4 t}$, Tammy Sage ${ }^{5}$ and Dean DellaPenna ${ }^{1 *}$ \\ 1 Department of Biochemistry and Molecular Biology, Michigan State University, East Lansing, MI, USA \\ ${ }^{2}$ Cell and Molecular Biology Program, Michigan State University, East Lansing, MI, USA \\ ${ }^{3}$ Department of Botany, University of Wisconsin-Madison, Madison, WI, USA \\ ${ }^{4}$ Genetics Program, Michigan State University, East Lansing, MI, USA \\ ${ }^{5}$ Department of Ecology and Evolutionary Biology, University of Toronto, Toronto, ON, Canada
}

Edited by:

Gregorio Hueros, Universidad de

Alcalá, Spain

Prem S. Chourey, United States

Department of Agriculture, USA

Paulo Monjardino, Universidade dos

Açores, Portugal

Jae-yean Kim, Gyeongsang National

University, South Korea

\section{*Correspondence:}

Dean DellaPenna, Department of Biochemistry and Molecular Biology,

Michigan State University, 2235

Molecular Plant Science, East

Lansing, MI 48824-1319, USA

e-mail:dellapen@msu.edu

equally to this work.

\section{Reviewed by:}

these authors have contributed

Tocopherols (vitamin E) are lipid-soluble antioxidants produced by all plants and algae, and many cyanobacteria, yet their functions in these photosynthetic organisms are still not fully understood. We have previously reported that the vitamin $E$ deficient 2 (vte2) mutant of Arabidopsis thaliana is sensitive to low temperature (LT) due to impaired transfer cell wall (TCW) development and photoassimilate export associated with massive callose deposition in transfer cells of the phloem. To further understand the roles of tocopherols in LT induced TCW development we compared the global transcript profiles of vte 2 and wild-type leaves during LT treatment. Tocopherol deficiency had no significant impact on global gene expression in permissive conditions, but significantly affected expression of 77 genes after $48 \mathrm{~h}$ of LT treatment. In vte 2 relative to wild type, genes associated with solute transport were repressed, while those involved in various pathogen responses and cell wall modifications, including two members of callose synthase gene family, GLUCAN SYNTHASE LIKE 4 (GSL4) and GSL11, were induced. However, introduction of gs/4 or gs/11 mutations individually into the vte2 background did not suppress callose deposition or the overall LT-induced phenotypes of vte2. Intriguingly, introduction of a mutation disrupting GSL5, the major GSL responsible for pathogen-induced callose deposition, into vte2 substantially reduced vascular callose deposition at LT, but again had no effect on the photoassimilate export phenotype of LT-treated vte2. These results suggest that GSL5 plays a major role in TCW callose deposition in LT-treated vte2 but that this GSL5dependent callose deposition is not the primary cause of the impaired photoassimilate export phenotype.

Keywords: tocopherols, transfer cells, callose synthase, Arabidopsis, sugar export, antioxidants, phloem parenchyma cells

\section{INTRODUCTION}

Tocopherols are essential nutrients in mammals and, together with tocotrienols, are collectively known as vitamin E (Evans and Bishop, 1922; Bramley et al., 2000; Schneider, 2005). As lipidsoluble antioxidants tocopherols quench singlet oxygen and scavenge lipid peroxyl radicals and hence terminate the autocatalytic chain reaction of lipid peroxidation (Tappel, 1972; Fahrenholtz et al., 1974; Burton and Ingold, 1981; Liebler and Burr, 1992; Kamal-Eldin and Appelqvist, 1996). Tocopherols are localized in biological membranes and associated with highly unsaturated fatty acids, and thus may also affect membrane properties, such as permeability and stability of membranes (Erin et al., 1984; Kagan, 1989; Stillwell et al., 1996; Wang and Quinn, 2000).

Tocopherols are synthesized only in photosynthetic organisms, including all plants and algae, and some cyanobacteria. However, tocopherol functions in these organisms remain poorly understood. The tocopherol-deficient vte2 (vitamin $e$ 2) mutant of Arabidopsis thaliana is defective in homogentisate phytyl transferase (HPT), the first committed enzyme of the pathway, and lacks all tocopherols and pathway intermediates (Collakova and DellaPenna, 2001; Savidge et al., 2002;
Sattler et al., 2004; Mene-Saffrane et al., 2010). The vte2 mutants exhibit reduced seed viability and defective seedling development associated with elevated lipid peroxidation (Sattler et al., 2004; Mene-Saffrane et al., 2010; DellaPenna and Mene-Saffrane, 2011), demonstrating that a primary role of tocopherols is to limit non-enzymatic lipid oxidation of polyunsaturated fatty acids (PUFAs), especially during seed desiccation and seedling germination. Transcript profiling studies further confirmed the importance of non-enzymatic lipid oxidation in triggering the oxidative and defense responses in germinating seeds of vte2 (Sattler et al., 2006).

In contrast to the drastic vte 2 seedling phenotype, the vte2 mutants that do survive early seedling development become virtually indistinguishable from wild type under permissive conditions and also under high light stress (Sattler et al., 2004; Maeda et al., 2006), suggesting that tocopherols are dispensable in mature plants even under highly photooxidative stress conditions. However, when tocopherol-deficient Arabidopsis plants are subjected to low temperature (LT) they developed a series of biochemical and physiological phenotypes (Maeda et al., 2006). As early as $6 \mathrm{~h}$ after LT treatment the vte2 mutants exhibit an 
impairment of photoassimilate export. This transport phenotype is accompanied by an unusual deposition of cell wall materials (i.e., callose) in the vasculature which likely creates a bottleneck for photoassimilate transport. Reduced photoassimilate export subsequently leads to carbohydrate and anthocyanin accumulation in source leaves, feedback inhibition of photosynthesis and ultimately growth inhibition of whole plants at LT (Maeda et al., 2006). This LT phenotype was independent of light level and was not associated with typical symptoms of photooxidative stress (i.e., photoinhibition, photobleaching, accumulation of zeaxanthin, or lipid peroxides) (Maeda et al., 2006).

The carbohydrate accumulation and callose deposition phenotypes of LT-treated vte 2 resemble the phenotypes of maize sucrose export defective 1 ( $s x d 1$ ) and potato SXD1-RNAi lines, which are also tocopherol deficient and accumulate carbohydrates without LT treatment (Russin et al., 1996; Provencher et al., 2001; Sattler et al., 2003; Hofius et al., 2004). Thus, a role for tocopherols in phloem loading is conserved among different plants with the unique, LT-inducibility of Arabidopsis vte2 mutant phenotype providing a useful tool to dissect the underlying mechanism. Detailed ultrastructure analysis of the vasculature of the Arabidopsis vte2 mutant during a LT time course revealed that callose deposition occurred before significant accumulation of carbohydrate and is restricted to the transfer cell wall (TCW) of phloem parenchyma cells adjacent to the companion cell/sieve element complex (Maeda et al., 2006). While the TCW is usually characterized by invaginated wall ingrowth toward the cytoplasm (Haritatos et al., 2000; Talbot et al., 2002; McCurdy et al., 2008), the phloem parenchyma cells of LT-treated vte 2 developed abnormally thickened TCW with irregular shaped ingrowths and massive callose deposition (Maeda et al., 2006). These results demonstrated that TCW-specific callose deposition is tightly linked with the defective photoassimilate export phenotype and is not a secondary effect caused by carbohydrate accumulation. However, the molecular mechanism underlying the callose deposition remains to be determined as does whether impaired phloem loading is due to vascular callose deposition in TCWs in the tocopherol-deficient mutants.

Analysis of membrane lipid composition in wild-type Arabidopsis and the vte 2 mutant during LT treatment further revealed that tocopherol deficiency in plastids alters the PUFA composition of endoplasmic reticulum (ER) derived membrane lipids prior to LT treatment (Maeda et al., 2008). Subsequently, mutations in FATTY ACID DESATURASE 2 (FAD2) and TRIGALACTOSYLDIACYLGLYCEROL 1 (TGD1), encoding the ER-localized oleate desaturase and the ER-to-plastid lipid ATPbinding cassette $(\mathrm{ABC})$ transporter, respectively, were identified as suppressors of the vte2 LT-induced phenotypes (sve loci) (Maeda et al., 2008; Song et al., 2010). These results provided biochemical and genetic evidence that alterations in extra-plastidic lipid metabolism are an upstream event in the initiation and development of the vte2 LT-induced phenotypes (Maeda et al., 2008; Song et al., 2010). The unexpected role of plastid-localized tocopherols in ER lipid metabolism has led to the recent discovery of a novel mechanism allowing biochemical continuity between the ER and chloroplast membranes (Mehrshahi et al., 2013). However, further investigation is required to understand the molecular links between tocopherol deficiency, lipid metabolism, and reduced photoassimilate export in LT-treated $v t e 2$.

In this study, microarray analysis of wild-type Arabidopsis and the vte2 mutant was used to investigate the effects of tocopherol deficiency on global gene expression at both permissive and LT conditions. While multiple studies have investigated transcriptome responses to vitamin E deficiency in animals (Barella et al., 2004; Rota et al., 2004, 2005; Nell et al., 2007; Oommen et al., 2007), no global gene expression profile of the effect of tocopherols in photosynthetic tissues has hitherto been undertaken in plants. Although almost no changes were observed in genome wide transcription between wild type and vte 2 under permissive conditions, 77 genes were identified as being differentially expressed in vte 2 compared to wild type in response to LT-treatment. Attempts to genetically suppress transfer cell callose deposition by introducing mutations for two GLUCAN SYNTHASE LIKE (GSL) genes, whose expression was strongly induced in LT treated $v t e 2$, or a mutation in GSL5, previously shown to be the primary GSL responsible for callose deposition in response to pathogen ingress, demonstrated that GSL5 is responsible for the majority of the LT-induced vasculature callose deposition in vte2. However, genetic elimination of this GSL5dependent callose deposition showed that it is not the direct cause of the LT-induced photoassimilate export phenotype of $v t e 2$.

\section{MATERIALS AND METHODS PLANT MATERIALS AND GROWTH CONDITIONS}

Arabidopsis plants were grown and treated at LT as described previously (Maeda et al., 2008). Briefly, seed were stratified for 4-7 days $\left(4^{\circ} \mathrm{C}\right)$, planted in an equal mixture of vermiculite, perlite, and soil with $1 \times$ Hoagland solution, and grown under permissive conditions: $12 \mathrm{~h}, 120 \mu \mathrm{mol}$ photon $\mathrm{m}^{-2} \mathrm{~s}^{-1}$ light at $22^{\circ} \mathrm{C} / 12 \mathrm{~h}$ darkness at $18^{\circ} \mathrm{C}$ and $70 \%$ relative humidity. Plants were watered every other day and with a half strength Hoagland solution once a week. For LT treatments, 4-week-old plants were transferred at the beginning of the light cycle to $12 \mathrm{~h}, 120 \mu \mathrm{mol}$ photon $\mathrm{m}^{-2}$ $\mathrm{s}^{-1}$ light $/ 12 \mathrm{~h}$ darkness at $7^{\circ} \mathrm{C}$. For microarray analysis, the 911 th oldest rosetta leaves from three independent plants were harvested together into a tube filled with liquid nitrogen $1 \mathrm{~h}$ into the light cycle after 48 and $120 \mathrm{~h}$ of LT-treatment or without LT-treatment (referred to as $0 \mathrm{~h}$ LT treatment).

RNA EXTRACTION, LABELING, AND HYBRIDIZATION FOR MICROARRAY

Total RNA was extracted using the RNAqueous RNA extraction kit and the Plant RNA Isolation Aid (Ambion) according to the manufacturer's instructions. Labeling and hybridization of RNA were conducted using standard Affymetrix protocols by the Michigan State University DNA Microarray Facility. ATH1 Arabidopsis GeneChips (Affymetrix, Santa Clara, CA) were used for measuring changes in gene expression levels. Total RNA was converted into cDNA, which was in turn used to synthesize biotinylated cRNA. The cRNA was fragmented into smaller pieces and then was hybridized to the GeneChips. After hybridization, the chips were automatically washed and stained with streptavidin phycoerythrin using a fluidics station. The chips were scanned by the GeneArray scanner at $570 \mathrm{~nm}$ emission and $488 \mathrm{~nm}$ excitation. 


\section{MICROARRAY DATA EVALUATION AND PREPROCESSING}

Raw chip data were analyzed with $\mathrm{R}$ software (version 2.9, http://www.r-project.org/). Because of various problems associated with mismatch (MM) probes (Bolstad et al., 2003; Irizarry et al., 2003), only perfect match (PM) probe intensities were used. To assess data quality, the AffyRNAdeg and QCReport functions in the simpleaffy package were used to generate the RNA degradation (Supplemental Figure S1) and quality control (QC) plots (Supplemental Figure S2) for all 18 chips. The Boxplot tool included in the affy and simpleaffy packages were used to investigate the data distribution of the 18 chips (Supplemental Figure S3). RMA function as implemented in the affy package was used for background adjustment, normalization and summarization. A cluster dendrogram (Figure 1) was generated by applying hclust function using average linkage clustering of Euclidean distance based on the normalized expression values from 18 chips.

\section{STATISTICAL ANALYSES FOR DIFFERENTIALLY EXPRESSED GENES}

Signal intensity data were analyzed with the use of a linear statistical model and an empirical Bayes method in the LIMMA package implemented in Bioconductor of R software (Smyth, 2005) to identify genes differentially expressed between genotypes at different time points of LT treatment. The $p$-values were adjusted for multiple testing with the Benjamini and Hochberg method to control the false discovery rate (Benjamini and Hochberg, 1995). Genes with adjusted $p$-values $<0.05$ were considered significant. A heat map of the 77 significantly different genes in $v t e 2$ at $48 \mathrm{~h}$ of LT treatment was generated using the average linkage clustering of Euclidean distance based on the normalized average expression values for each genotype and timepoint.

The 49 genes that were significantly induced in $48 \mathrm{~h}$ LT-treated vte 2 relative to wild-type (Col) plants (Table 1)

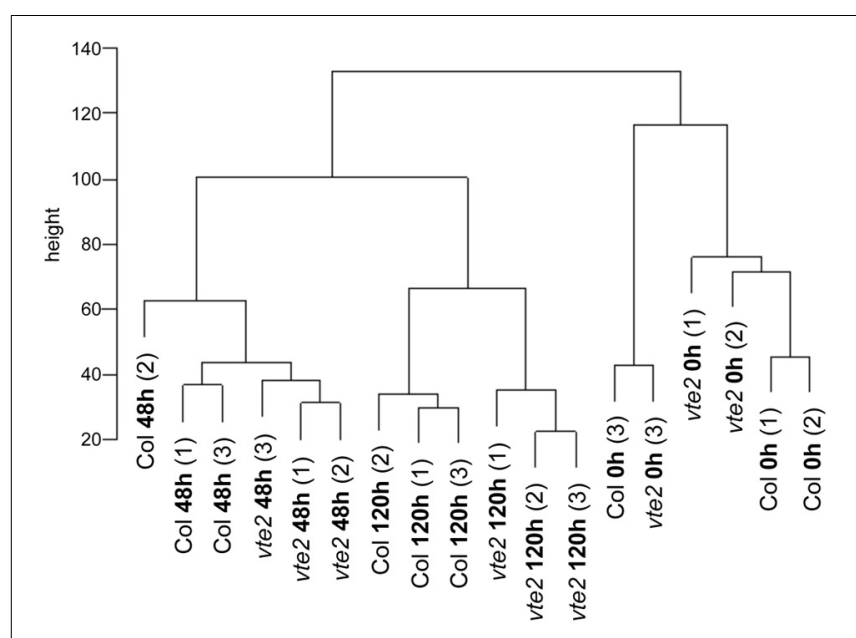

FIGURE 1 | A cluster dendrogram of a correlation matrix for all-against-all chip comparisons. The scale on the vertical bar "height" indicates Manhattan distance. The numbers (1) to (3) indicate three independent biological replications for treatments and genotypes. The samples cluster by different genotypes ( $\mathrm{Col}$ and vte2) after LT treatment (48 and $120 \mathrm{~h}$ ). were also examined using the Meta-Analyzer feature of GENEVESTIGATOR (Zimmermann et al., 2004) to assess their responses to various conditions or treatments. The gene expression responses were calculated as ratios between a given treatment and its negative control with the resulting values reflecting up- or down-regulation of genes by a treatment. Twelve different stress conditions were chosen: Biotic stress treatments with Botrytis cinerea (6 treatment chips +6 control chips), Pseudomonas syringae $(3+3)$, and Myzus persicae $(3+3)$. Chemical stress treatments were hydrogen peroxide $\left(\mathrm{H}_{2} \mathrm{O}_{2}\right)(3+2)$ and ozone $(3+3)$. Hormone stress treatments were with abcisic acid (ABA, $2+2)$, ethylene $(3+3)$, indole acidic acid (IAA, $3+3)$, and zeatin $(3+3)$. Abiotic stress treatments were cold $(3+3)$, drought $(3+1)$, and heat $(2+2)$. All experiments selected from GENEVESTIGATOR utilized mature leaves except for the hormone treatment data sets (ethylene, IAA, and zeatin) that were performed on seedlings. In addition, to compare the transcriptional responses to tocopherol deficiency in seedlings and mature plants (Table 3), microarray data for 0-day and 3-dayold seedlings of Col and vte2 (three biological replicates for each treatment and genotype, see Sattler et al., 2006) were subjected to the same procedure of preprocessing and statistical analysis as described above for vte 2 plants under LT treatment.

\section{GENERATION OF MUTANT GENOTYPES}

The following $g s l$ mutant lines were obtained from the Arabidopsis Biological Resource Center at Ohio State University: SALK_000507 for GSL4 (with a T-DNA insert in exon 34 of At3g14570), gsl5-1/pmr4-1 for GSL5 (with a non-sense mutation in exon 2 of At4g03550), and SALK_019534 for GSL11 (with a T-DNA insert in exon 15 of At3g59100). Homozygous mutant lines for gsl5-1 were identified by PCR using CAPs genotyping primers (Nishimura et al., 2003). Homozygous mutants for gsl4 and gsll1 were identified by PCR using gene specific primers; $5^{\prime}$ - TTGCCTGAGAGGATTAGCAAG - $3^{\prime}$ (forward) and $5^{\prime}$-TTGAAGGATACAAGGACGTGG - $3^{\prime}$ (reverse) for $g s l 4$ and $5^{\prime}$-TCACACCTTCATTCCCTGTTC $-3^{\prime}$ (forward) and $5^{\prime}$-GTTCCTGTGTAAGGCCTCATG $-3^{\prime}$ (reverse) for $g s l 11$. The double homozygous mutant genotypes vte2 gsl4, vte2 gsl5, and vte 2 gsl11 were obtained by HPLC analysis for tocopherol deficiency (Collakova and DellaPenna, 2001) and the above mentioned PCR-based genotyping for gsl homozygosity. vte2 homozygosity was also confirmed by a CAPs marker developed for the vte2-1 point mutation (Maeda et al., 2006). Plants of Col, vte2, the single mutants of gsl4, gsl5, gsl11, and the three double mutants were grown for 4 weeks at permissive conditions and then transferred to LT conditions for the time periods, indicated in each figure legend for evaluation of different LT-induced phenotypes. Optimal time points were chosen based on our previous time-course analysis of the appearance of different LT-induced vte2 phenotypes (Maeda et al., 2006, 2008).

\section{${ }^{14}$ C PHOTOASSIMILATE LABELING AND ANALYSIS OF SUGARS}

Analyses for leaf glucose, fructose, and sucrose levels were performed as previously described (Maeda et al., 2006). ${ }^{14} \mathrm{CO}_{2}$ labeling of photoassimilate and measurement of phloem 
Table 1 | The 49 genes significantly upregulated in vte2 relative to Col at $48 \mathrm{~h}$ of LT treatment.

\begin{tabular}{|c|c|c|c|c|}
\hline AGI number & $\mathbf{M}^{\mathbf{a}}$ & adj-P.value ${ }^{b}$ & $\mathbf{B}^{\mathbf{c}}$ & Annotated gene function ${ }^{d}$ \\
\hline At4g23410 & 1.53 & 0.00 & 10.08 & Senescence-associated family protein \\
\hline At5g22860 & 1.38 & 0.00 & 7.04 & Serine carboxypeptidase S28 family protein \\
\hline At3g14570 & 1.85 & 0.00 & 6.88 & Glycosyl transferase family 48 protein (glucan synthase like 4, GSL4) \\
\hline At1g74590 & 1.50 & 0.00 & 6.31 & Glutathione S-transferase, putative \\
\hline At4g20320 & 1.08 & 0.00 & 5.80 & CTP synthase, putative/UTP-ammonia ligase, putative \\
\hline At3g22910 & 1.82 & 0.00 & 5.65 & Ca-transporting ATPase, plasma membrane-type, putative (ACA13) \\
\hline At5g13080 & 1.88 & 0.00 & 5.51 & WRKY family transcription factor (WRKY75) \\
\hline At5g47920 & 1.06 & 0.00 & 5.13 & Expressed protein \\
\hline At1g74055 & 1.00 & 0.00 & 4.18 & Expressed protein \\
\hline At1g30370 & 1.60 & 0.01 & 3.80 & Lipase class 3 family protein \\
\hline At5g13880 & 1.45 & 0.01 & 3.60 & Expressed protein \\
\hline At3g49130 & 0.94 & 0.01 & 3.16 & Hypothetical protein \\
\hline At5g46590 & 1.31 & 0.01 & 3.09 & No apical meristem (NAM) family protein \\
\hline At3g21780 & 0.91 & 0.01 & 3.09 & UDP-glucosyl transferase family protein \\
\hline At1g17180 & 1.21 & 0.02 & 2.82 & Glutathione S-transferase, putative \\
\hline At1g65610 & 1.33 & 0.02 & 2.70 & Endo-1,4-beta-glucanase, putative/cellulase, putative \\
\hline At3g53600 & 0.70 & 0.02 & 2.39 & Zinc finger ( $\mathrm{C} 2 \mathrm{H} 2$ type) family protein \\
\hline At5g13170 & 1.01 & 0.02 & 2.37 & Nodulin MtN3 family protein \\
\hline At3g59100 & 1.04 & 0.03 & 1.81 & Glycosyl transferase family 48 protein (glucan synthase like 11, GSL11) \\
\hline At4g19460 & 0.80 & 0.03 & 1.80 & Glycosyl transferase family 1 protein \\
\hline At3g09270 & 2.07 & 0.03 & 1.73 & Glutathione S-transferase, putative \\
\hline At5g22570 & 1.18 & 0.03 & 1.72 & WRKY family transcription factor (WRKY38) \\
\hline At1g32350 & 1.45 & 0.04 & 1.64 & Alternative oxidase, putative \\
\hline At5g17330 & 0.74 & 0.04 & 1.54 & Glutamate decarboxylase 1 (GAD 1) \\
\hline At4g28550 & 1.05 & 0.04 & 1.43 & RabGAP/TBC domain-containing protein \\
\hline At5g65600 & 1.27 & 0.04 & 1.38 & Legume lectin family protein/protein kinase family protein \\
\hline At4g36430 & 0.79 & 0.04 & 1.37 & Peroxidase, putative \\
\hline At5g04080 & 0.63 & 0.04 & 1.32 & Expressed protein \\
\hline At5g64905 & 1.78 & 0.04 & 1.25 & Expressed protein \\
\hline At5g66920 & 1.09 & 0.04 & 1.24 & Multi-copper oxidase type I family protein \\
\hline At5g63970 & 0.97 & 0.05 & 1.20 & Copine-related \\
\hline At2g23270 & 0.95 & 0.05 & 1.20 & Expressed protein \\
\hline At1g19250 & 0.86 & 0.05 & 1.10 & Flavin-containing monooxygenase family protein \\
\hline At5g67080 & 1.69 & 0.05 & 1.08 & Protein kinase family protein \\
\hline
\end{tabular}

${ }^{a} M$-value $(M)$ is the value of the contrast and represents a $\log _{2}$ fold change between 48 h-LT-treated vte2 and Col.

badj-Pvalue is the p-value adjusted for multiple testing with Benjamini and Hochberg's method to control the false discovery rate.

${ }^{c} B$-statistic $(B)$ is the log-odds that the gene is differentially expressed.

dAnnotation was obtained from the Gene Ontology of The Arabidopsis Information Resources. 
exudation were also carried out as described (Maeda et al., 2006) except that $10 \mathrm{mM}$ EDTA was used for exudation buffer and $0.05 \mathrm{mCi}$ of $\mathrm{NaH}^{14} \mathrm{CO}_{3}$ was used per labeling experiment. Phloem exudates were collected after $5 \mathrm{~h}$ of exudation.

\section{FLUORESCENCE AND TRANSMISSION ELECTRON MICROSCOPY}

Leaves were prepared for aniline blue fluorescence microscopy and staining and visualization were performed as described (Maeda et al., 2006) except that the gain adjustment of the camera was set to 2.0 for images in Figures 4B, 5A. Leaves were prepared for transmission electron microscopy and immunolocalization of $\beta$-1,3-glucan as described (Maeda et al., 2006).

\section{RESULTS}

\section{TOCOPHEROL DEFICIENCY HAS LITTLE IMPACT ON GLOBAL GENE EXPRESSION AT PERMISSIVE CONDITIONS}

To identify changes in gene expression that might be specifically related to the absence of tocopherols, global transcript profiles were compared between vte 2 and Col plants grown under permissive conditions for 4 weeks, when they are physiologically and biochemically indistinguishable, and at two time points of LT treatment (48 and $120 \mathrm{~h}$ ) selected based on our previous timecourse study of the physiological and biochemical changes of vte 2 and Col during LT treatment (Maeda et al., 2006). After $48 \mathrm{~h}$ of LT, vascular callose deposition is strongly induced and photoassimilate export capacity is significantly lower in vte 2 compared to Col, though the visible whole plant phenotypes and soluble sugar accumulation between the two genotypes do not differ (Maeda et al., 2006). The $120 \mathrm{~h}$ LT timepoint represents a relatively late response time point when soluble sugars are significantly higher and callose deposition is even more extensive and wide spread in vte2 (Maeda et al., 2006). Thus the $48 \mathrm{~h}$ time point should allow identification of early responses to tocopherol deficiency that are distinct from later, pleiotropic responses resulting from the strongly elevated sugar levels in $v t e 2$ after $120 \mathrm{~h}$ of LT. The $0 \mathrm{~h}$ time point represents the absence of LT treatment (see Materials and Methods) and serves as a critical treatment control.

The vte 2 LT experiment comprised 18 chips in a factorial design. Three independent biological replicates were conducted for Col and vte 2 at each of the three time points, allowing rigorous statistical analysis of the data obtained. When the relationship of chips was examined by a cluster dendrogram, three clusters consistent with the three time points of LT treatment were apparent (Figure 1). The 48 and $120 \mathrm{~h}$ LT-treated samples were more closely related to each other than to the $0 \mathrm{~h}$ data, suggesting that the effect of LT treatment was greater than effects due to genotypic differences.

To investigate if tocopherol deficiency leads to any transcriptional changes before LT treatment, the linear models for microarray data (limma) analysis (Smyth, 2005) was performed to detect differently expressed genes between vte2 and Col at $0 \mathrm{~h}$ (see Materials and Methods). With the exception of At2g18950, which encodes the mutated gene in the background (VTE2/HPT, Collakova and DellaPenna, 2003), no other statistically significant differences (at adjusted $p$-values of $<0.05$ ) were observed. These data indicate that the lack of tocopherols per se has little impact on global gene expression in mature $v t e 2$ plants under permissive conditions.

\section{IDENTIFICATION OF DIFFERENTIALLY EXPRESSED GENES IN $48 \mathrm{~h}$-LT-TREATED vte2 AND Col}

After $48 \mathrm{~h}$ at LT, 77 probe sets were found to be significantly different between vte 2 and Col: 49 genes were significantly induced (Table 1) and 28 were significantly repressed (Table 2 ) in vte2 relative to Col. The expression patterns of these 77 genes across all time points are visualized in the gene tree in Figure 2. As discussed above, before LT treatment $(0 \mathrm{~h})$ expression levels of all genes are very similar between $\mathrm{Col}$ and vte 2 and changed differently between genotypes after LT treatment. Group I contains 43 genes whose expression is generally low at $0 \mathrm{~h}$ and induced in both Col and vte2 after LT treatment, with induction in vte2 being stronger and more persistent. Group II contains 17 genes whose expression is somewhat high at $0 \mathrm{~h}$ and then more strongly induced or repressed in vte 2 after LT treatment compared to Col. Group III (12 genes) and IV ( 5 genes) are expressed at moderately and very high levels at $0 \mathrm{~h}$, respectively, and both repressed at LT more strongly and persistently in vte2 than Col. Several genes in groups I, II, and III show opposite expression patterns in vte 2 and Col from 0 to $48 \mathrm{~h}$ of LT treatment (highlighted in red for induced or blue for repressed in vte2 relative to Col, respectively) and are particularly interesting as they represent potential "marker genes" that are specifically impacted by tocopherol deficiency at LT.

Among the 49 induced genes at $48 \mathrm{~h}$ (Table 1), 6 are annotated as glycosyl transferases (At3g14570, At3g59100, At4g19460), UDP-glucosyl transferase (At3g21780), or glucanases (At1g26450, At1g65610). These genes are likely involved in aspects of cell wall modification, consistent with the major modifications to cell wall structure in phloem parenchyma cells of LT-treated vte2 (Maeda et al., 2006, 2008). Notably, LT treatment induced significant, albeit low, expression of two putative callose synthase genes, GSL4 (At3g14570) and GSL11 (At3g59100) in vte2 (Table 1). These genes are two members of the 12 member GSL callose synthase gene family in Arabidopsis and may contribute to the substantial callose deposition in transfer cells of LT-treated vte2. Other notable upregulated genes in LT-treated vte 2 are involved in stress and senescence responses, various signaling pathways, and transcriptional regulation, including WRKY (At5g13080, At5g46350, At5g22570), NAM (At5g46590), and zinc finger $\left(\mathrm{C}_{2} \mathrm{H}_{2}\right.$ type, At3g53600) transcription factors (Table 1).

The most significantly repressed gene in $v t e 2$ at $48 \mathrm{~h}$ LT (Table 2) was VTE2/HPT (At2g18950, Collakova and DellaPenna, 2003), the locus mutated in the vte2 background. Other downregulated genes of interest include a methionine sulfoxide reductase (At4g04830, EC 1.8.4.6), one member of a small gene family encoding enzymes that reduce oxidized methionine residues of proteins, and four genes encoding nodulin drug/metabolite transporters (Table 2). Nodulins are involved in nodulation of legume roots during symbiosis with Rhizobia, a process where extensive metabolite transport across peribacteroid membranes is required (Vandewiel et al., 1990; Hohnjec et al., 2009). Repression of these nodulins might be related to altered carbohydrate transport in LT-treated $v$ te 2 . 
Table 2 | The 28 genes significantly downregulated in vte2 relative to Col at $48 \mathrm{~h}$ of LT treatment.

\begin{tabular}{|c|c|c|c|c|}
\hline AGI number & $\mathbf{M}^{\mathbf{a}}$ & adj-P.value ${ }^{b}$ & $\mathbf{B}^{\mathbf{c}}$ & Annotated gene function ${ }^{d}$ \\
\hline At2g18950 & -2.36 & 0.00 & 7.93 & HPT: tocopherol phytyltransferase \\
\hline At5g14740 & -0.77 & 0.00 & 6.32 & Carbonate dehydratase 2 (CA2) (CA18) \\
\hline At3g11930 & -1.47 & 0.00 & 6.10 & Universal stress protein (USP) family protein \\
\hline At2g36830 & -1.01 & 0.00 & 5.94 & Major intrinsic family protein/MIP family protein \\
\hline At1g04680 & -0.74 & 0.00 & 5.41 & Pectate lyase family protein \\
\hline At1g76800 & -1.43 & 0.00 & 5.36 & Nodulin, putative \\
\hline At4g08300 & -2.98 & 0.00 & 4.58 & Nodulin MtN21 family protein \\
\hline At2g22330 & -1.67 & 0.00 & 4.44 & Cytochrome P450, putative \\
\hline At3g10080 & -0.72 & 0.01 & 3.86 & Germin-like protein, putative \\
\hline At5g44720 & -1.05 & 0.02 & 2.89 & Molybdenum cofactor sulfurase family protein \\
\hline At5g23020 & -3.81 & 0.02 & 2.88 & 2-isopropylmalate synthase 2 (IMS2) \\
\hline At3g47470 & -0.84 & 0.02 & 2.77 & Chlorophyll A-B binding protein 4, chloroplast/LHCl type III CAB-4 (CAB4) \\
\hline At1g51400 & -0.88 & 0.02 & 2.61 & Photosystem II 5 kD protein \\
\hline At4g08290 & -1.22 & 0.02 & 2.56 & Nodulin MtN21 family protein \\
\hline At1g21440 & -1.17 & 0.03 & 2.18 & Mutase family protein \\
\hline At1g01620 & -0.83 & 0.03 & 2.02 & $\begin{array}{l}\text { Plasma membrane intrinsic protein 1C } \quad \text { (PIP1C)/aquaporin PIP1.3 } \\
\text { (PIP1.3)/transmembrane protein } \mathrm{B} \text { (TMPB) }\end{array}$ \\
\hline At3g08940 & -0.82 & 0.03 & 1.95 & Chlorophyll A-B binding protein (LHCB4.2) \\
\hline At5g24490 & -1.22 & 0.03 & 1.91 & 30 S ribosomal protein, putative \\
\hline At4g04830 & -1.57 & 0.04 & 1.58 & Methionine sulfoxide reductase domain-containing protein \\
\hline At2g37460 & -1.93 & 0.04 & 1.54 & Nodulin MtN21 family protein \\
\hline At4g04040 & -0.69 & 0.04 & 1.44 & $\begin{array}{l}\text { Pyrophosphate-fructose-6-phosphate 1-phosphotransferase beta sub- } \\
\text { unit, putative }\end{array}$ \\
\hline At1g31180 & -0.85 & 0.04 & 1.41 & 3-isopropylmalate dehydrogenase, chloroplast, putative \\
\hline At1g78370 & -1.07 & 0.04 & 1.40 & Glutathione S-transferase, putative \\
\hline At5g02260 & -1.23 & 0.04 & 1.38 & Expansin, putative (EXP9) \\
\hline At5g67070 & -0.51 & 0.04 & 1.28 & Rapid alkalinization factor (RALF) family protein \\
\hline At1g13280 & -0.94 & 0.04 & 1.26 & Allene oxide cyclase family protein \\
\hline At3g09580 & -0.73 & 0.05 & 1.08 & Amine oxidase family protein \\
\hline At1g03600 & -0.48 & 0.05 & 1.08 & Photosystem II family protein \\
\hline
\end{tabular}

${ }^{a} M$-value $(M)$ is the value of the contrast and represents a $\log _{2}$ fold change between 48 h-LT-treated vte2 and Col.

${ }^{b}$ adj-P.value is the p-value adjusted for multiple testing with Benjamini and Hochberg's method to control the false discovery rate.

${ }^{c} B$-statistic $(B)$ is the log-odds that the gene is differentially expressed.

${ }^{d}$ Annotation was obtained from the Gene Ontology of The Arabidopsis Information Resources.

\section{THE 49 GENES INDUCED IN vte2 ARE NOT UPREGULATED BY ABIOTIC STRESS per se}

To investigate whether the 49 genes induced in LT-treated vte2 are part of a general stress response their expression patterns under diverse abiotic and biotic stress conditions were examined using publically available Arabidopsis microarray data (Figure 3 ). Approximately half of the 49 genes induced in LT-treated vte2 were also strongly and specifically upregulated by biotic treatments including the necrotrophic fungus Botrytis cinerea and pathogenic leaf bacterium Pseudomonas syringae but not the phloem-feeding aphid Myzus persicae. Interestingly, many of the genes that were upregulated by pathogen treatments were also induced by ozone treatment (Figure 3). These include most of the transcription factors (WRKY, NAM, and zinc finger family proteins) and some of the stress- and signaling-related genes (Glutathione S-transferases: At1g74590, At1g17180, At3g09270; auxin-responsive GH3 family proteins: At1g59500, At4g27260) induced in LT-treated $v t e 2$. In contrast, very few genes induced in LT-treated vte2 overlapped with genes responsive to abiotic stress conditions including heat, drought, and cold treatments or hormone treatments such as zeatin, IAA, ethylene, or ABA (Figure 3). These results suggest that the 49 genes with induced expression in LT-treated $v t e 2$ are due to tocopherol deficiency rather than a general response to cold or other abiotic stresses.

A prior study showed that the transcriptome of germinating vte2 seedlings (at permissive conditions) is substantially influenced by elevated non-enzymatic lipid peroxidation occurring in the mutant at this developmental stage (Sattler et al., 2006). To compare transcriptional responses of LTtreated vte2 with those of 3-day-old vte2 seedlings, our preprocessing and statistical analysis approach was applied to germinating $\mathrm{Col}$ and vte 2 seedling microarray datasets. Out of 744 genes identified as significantly different (adjusted $p<$ 0.05) in 3-day-old vte2 seedlings relative to 3-day-old Col, only 12 were in common with the 77 significant altered 


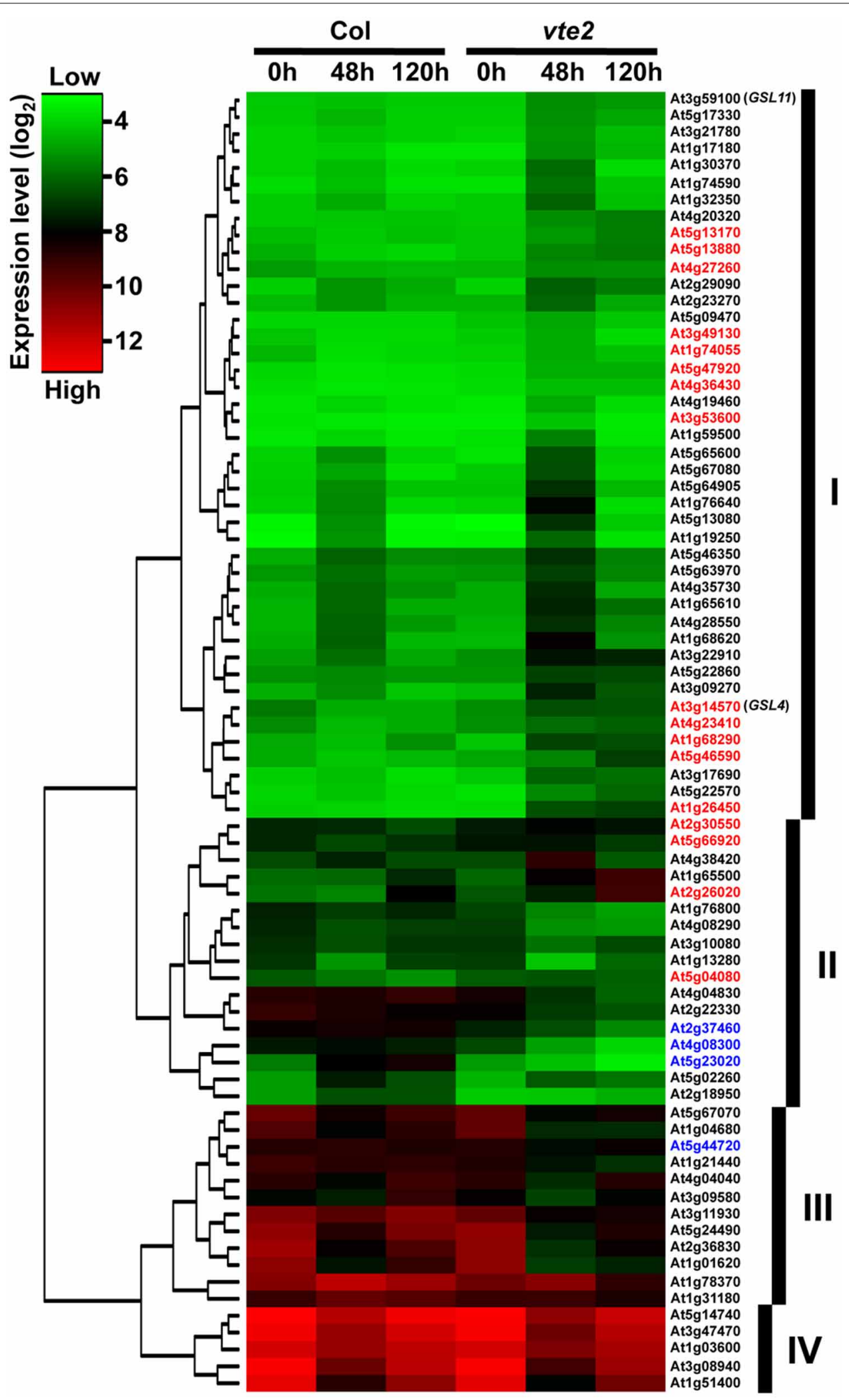

FIGURE 2 | A gene tree of the $\mathbf{7 7}$ genes significantly altered in 48 h-LT-treated vte2 relative to $\mathrm{Col}$ (adjusted $\boldsymbol{p}$-value $<\mathbf{0 . 0 5}$ ). The color bar represents expression levels $\left(\log _{2}\right)$, with green to red being low to high expression. The groups (labeled as I, II, III, and IV) were based on general expression patterns across $\mathrm{Col}$ and vte 2 at three time points of LT treatment Genes showing opposite directions of expression from 0 to $48 \mathrm{~h}$ of LT treatment in Col and vte2 are highlighted in red (induced in vte2) or blue (repressed in vte2). See text for additional details. 

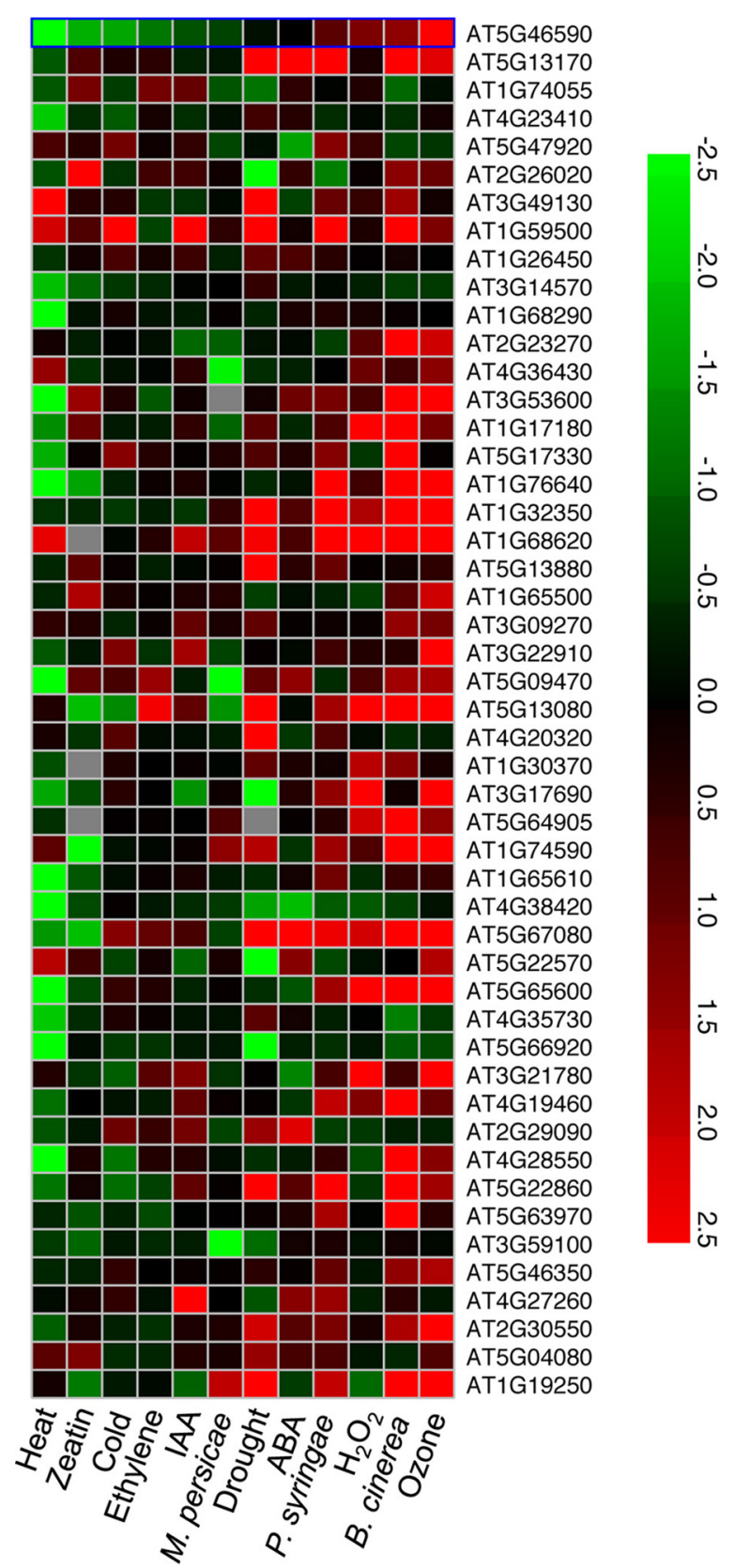

FIGURE 3 | A heat map of expression patterns for the 49 upregulated genes in $48 \mathrm{~h}$ LT-treated vte2 under other stress conditions. Genes (AG) number) significantly induced in $48 \mathrm{~h} \mathrm{LT-treated} \mathrm{vte2} \mathrm{relative} \mathrm{to} \mathrm{Col} \mathrm{plants}$ were examined using the Meta-Analyzer feature of GENEVESTIGATOR to assess their responses to various stress conditions or treatments. The conditions and treatments shown were selected from GENEVESTIGATOR to represent a range of biotic, abiotic, and major hormone treatments and are sorted by increasing ratios from the left to the right. The color bar represents expression levels $\left(\log _{2}\right)$ relative to each corresponding negative control in GENEVESTIGATOR.

genes in 48 h-LT-treated vte2 (Table 3 ). These results suggest that the majority of the transcriptional response of LT-treated vte2 plants is largely distinct from that of vte 2 seedlings.

\section{gs/4 AND gs/11 HAVE LITTLE IMPACT ON LT-INDUCED vte2 CALLOSE} DEPOSITION

Vascular-specific callose deposition is a phenotype shared by tocopherol-deficient mutants in several plant species (Russin et al., 1996; Hofius et al., 2004; Maeda et al., 2006). It has been suggested that vascular-specific callose deposition may directly block photoassimilate translocation and lead to the subsequent carbohydrate accumulation and growth inhibition phenotypes in tocopherol-deficient plants (Russin et al., 1996; Hofius et al., 2004; Maeda et al., 2006). To test this hypothesis we attempted to genetically eliminate induced callose deposition in vte 2 by introducing mutations affecting specific callose synthase genes. Among the 12 GSL genes encoding putative callose synthases in the Arabidopsis genome (Richmond and Somerville, 2000; Hong et al., 2001), GSL4 (At3g14570) and GSL11 (At3g59100) were the only two family members whose expression was significantly altered in both 48 and $120 \mathrm{~h}$ LT-treated vte2 relative to Col (adjusted $p$-value $<0.01$ ) (Table 1, Supplemental Figure S5). Homozygous mutants of GSL4 and GSL11 were therefore selected and introduced into the vte 2 background (see Materials and Methods) and the single gsl and vte2 mutants and gsl 4 vte 2 and gsl11 vte2 double mutants were subjected to LT treatment to assess their phenotypes. Before LT treatment, the visible phenotypes of all the single and double mutants were similar to Col (Figure 4A). After prolonged LT treatment (28 days), which allows for full development of visible vte2 LT phenotypes (Maeda et al., 2006), the gsl4 and gsll1 mutants appeared similar to Col while both of the double mutants were smaller, purple and similar to vte 2 (Figure 4A), indicating that neither the gsl4 nor gsl11 mutations have a substantial impact on the vte2 LT phenotype. The levels of LT-induced vascular callose deposition (an early phenotype, as described in Maeda et al., 2006) detectable by aniline-blue fluorescence after 3 days LT treatment was also indistinguishable between the vte 2 single mutant and the gsl 4 te 2 or gsl11 vte 2 double mutants (Figure 4B). Thus, although GSL4 and GSL11 transcript levels are the only GSL family members induced higher in vte 2 than $\mathrm{Col}$ in response to LT treatment, loss of either gene activity does not have a major impact on callose deposition in LT-treated $v t e 2$.

\section{gs/5 ATTENUATES THE MAJORITY OF CALLOSE DEPOSITION IN vte2 WITHOUT SUPPRESSING THE PHOTOASSIMILATE EXPORT PHENOTYPE}

Given that callose synthases are often post-transcriptionally regulated (Zavaliev et al., 2011), it is possible that enzymes responsible for the vascular-specific callose deposition of LT-treated vte 2 may be post-transcriptionally induced and not identified as differentially expressed between vte2 and Col. GSL5 is the best characterized of the 12 GSLs in Arabidopsis and has been shown to be required for callose formation in response to wounding and fungal pathogens (Jacobs et al., 2003; Nishimura et al., 2003). Although GSL5 expression is modestly induced in response to LTtreatment and not differentially expressed in LT-treated $v$ te 2 relative to wild type (Table 1, Supplemental Figure S5), it might still play a role in the vascular callose deposition of vte2. To test this possibility we introduced the gsl5 mutation into the vte2 background and examined its effect on LT-induced vte 2 phenotypes, 
Table 3 | The 12 genes that are common between the 77 significantly different genes in 48 h-LT-treated vte2 plant and 744 significantly different genes in 3-d-old vte2 seedling.

\begin{tabular}{|c|c|c|c|c|c|c|c|}
\hline \multirow[t]{2}{*}{$\begin{array}{l}\text { AGI } \\
\text { number }\end{array}$} & \multicolumn{2}{|c|}{$\begin{array}{l}48 \text { h-LT-treated } \\
\text { vte2 plant }\end{array}$} & \multicolumn{2}{|c|}{$\begin{array}{l}\text { 3-day-old vte2 } \\
\text { seedling }\end{array}$} & \multirow[t]{2}{*}{ Gene title ${ }^{c}$} & \multirow[t]{2}{*}{ GO molecular function ${ }^{\mathrm{c}}$} & \multirow[t]{2}{*}{ GO cellular component ${ }^{c}$} \\
\hline & $\mathbf{M}^{\mathbf{a}}$ & adj-P.value ${ }^{b}$ & $\mathbf{M}^{\mathbf{a}}$ & adj-P.value ${ }^{b}$ & & & \\
\hline At1g68290 & 2.39 & 0.00 & 2.01 & 0.00 & $\begin{array}{l}\text { Bifunctional nuclease, } \\
\text { putative }\end{array}$ & $\begin{array}{l}\text { Nucleic acid } \\
\text { binding/endonuclease } \\
\text { activity }\end{array}$ & Endomembrane system \\
\hline At5g46590 & 1.31 & 0.01 & 1.93 & 0.00 & $\begin{array}{l}\text { No apical meristem } \\
\text { (NAM) family protein }\end{array}$ & $\begin{array}{l}\text { Transcription factor } \\
\text { activity/DNA binding }\end{array}$ & - \\
\hline At1g17180 & 1.21 & 0.02 & 1.93 & 0.03 & Glutathione S-transferase & $\begin{array}{l}\text { Glutathione transferase } \\
\text { activity }\end{array}$ & Cytoplasm \\
\hline At2g30550 & 0.07 & 0.03 & 1.02 & 0.05 & $\begin{array}{l}\text { Lipase class } 3 \text { family } \\
\text { protein }\end{array}$ & $\begin{array}{l}\text { Triacylglycerol lipase } \\
\text { activity }\end{array}$ & Chloroplast \\
\hline At4g36430 & 0.79 & 0.04 & 3.20 & 0.00 & Peroxidase, putative & $\begin{array}{l}\text { Peroxidase } \\
\text { activity/calcium ion } \\
\text { binding/oxidoreductase } \\
\text { activity }\end{array}$ & Endomembrane system \\
\hline At5g64905 & 1.78 & 0.04 & 1.10 & 0.01 & Expressed protein & - & - \\
\hline At1g76800 & -1.43 & 0.00 & -0.74 & 0.02 & Nodulin, putative & - & - \\
\hline At3g09580 & -0.73 & 0.05 & -0.90 & 0.02 & $\begin{array}{l}\text { Amine oxidase family } \\
\text { protein }\end{array}$ & Oxidoreductase activity & Chloroplast \\
\hline
\end{tabular}

${ }^{a} M$-value $(M)$ is the value of the contrast and represents a $\log _{2}$ fold change.

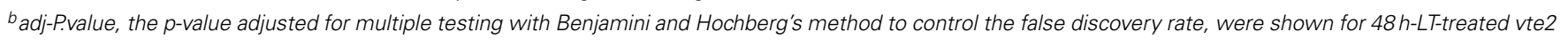
plant and 3-d-old vte2 seedling, respectively.

${ }^{c}$ Descriptions of gene function and cellular component were obtained from the Gene Ontology section of The Arabidopsis Information Resources.

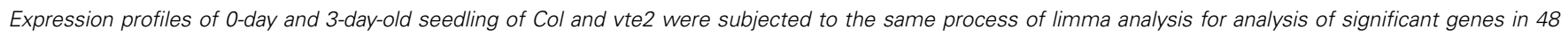

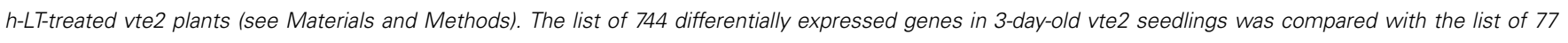
differentially expressed genes in 48 h-LT-treated vte2 plants and overlapping 12 genes were listed.

including vascular callose deposition. Under permissive conditions, the gsl5 vte2 double mutant had a visible phenotype similar to Col, gsl5 and vte2 (Supplemental Figure S4). When 4 week-old plants were subjected to 7 days of LT treatment (which induces a stronger callose deposition phenotype than 3 days of LT treatment), vte 2 exhibited the expected strong vascular-specific callose deposition, while no fluorescence signal was detectable in the vasculature of $\mathrm{Col}$ and $g s l 5$ (Figure 5A). Although the gsl5 vte 2 double mutant showed a substantial reduction in fluorescence intensity, weakly fluorescent spots were still present in the gsl5 vte2 vasculature (Figure 5A). However, despite the strong reduction in callose deposition at LT, photoassimilate export capacity, elevated soluble sugar content, and the visible phenotype of $g s l 5$ vte 2 were indistinguishable from that of vte2 (Figures 5B-D).

To further address the role of the remaining GSL5independent callose deposition in gsl5 vte2, transmission electron microscopy was used to examine the ultrastructure and localization of callose in TCWs of Col, gsl5, vte2, and gsl5 vte2. The spatial organization and types of cells constituting the phloem and xylem of all genotypes were similar to prior reports (Haritatos et al., 2000; Maeda et al., 2006) and notable differences were observed only in the phloem parenchyma TCWs following 3 days of LT treatment. In all instances, cell wall differentiation ensued in phloem transfer cells of LT-treated plants. Both Col and gsl5 developed uniform TCWs adjacent to the companion cell/sieve element complex (Figures 6A,B,E,F), whereas those in vte 2 and gsl5 vte 2 were not uniform and were abnormally thickened to varying degrees (Figures 6C,D,G-I). The extensive localized globular outgrowths of wall commonly found in transfer cells of vte2 (Maeda et al., 2006) also developed in gsl5 vte2 but were less frequent. Positive immunolocalization with monoclonal antibodies to callose ( $\beta$-1,3-glucan) showed it was present in TCWs at the companion cell/sieve element boundary in vte2 (Maeda et al., 2006), and also in gsl5 vte2 (Figures 6H,I). Immunolabeling of 


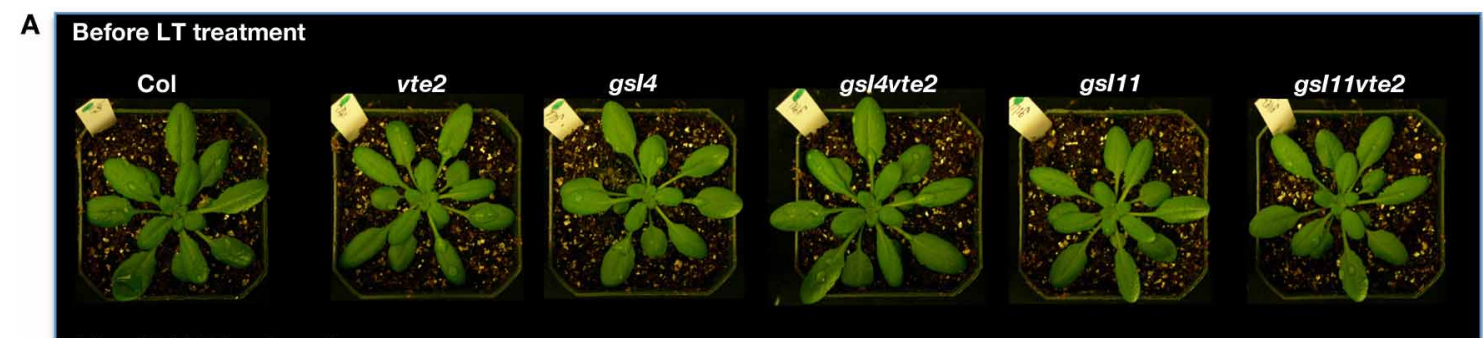

After 14d LT treatment

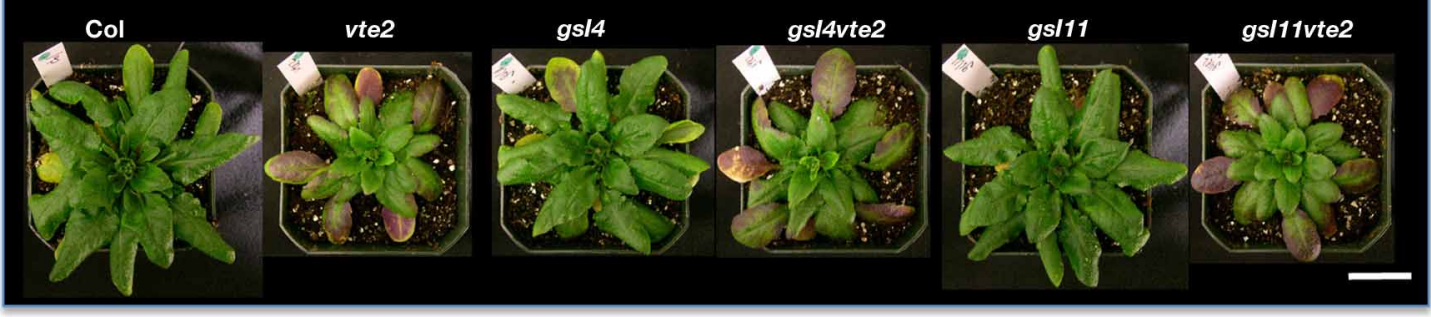

B
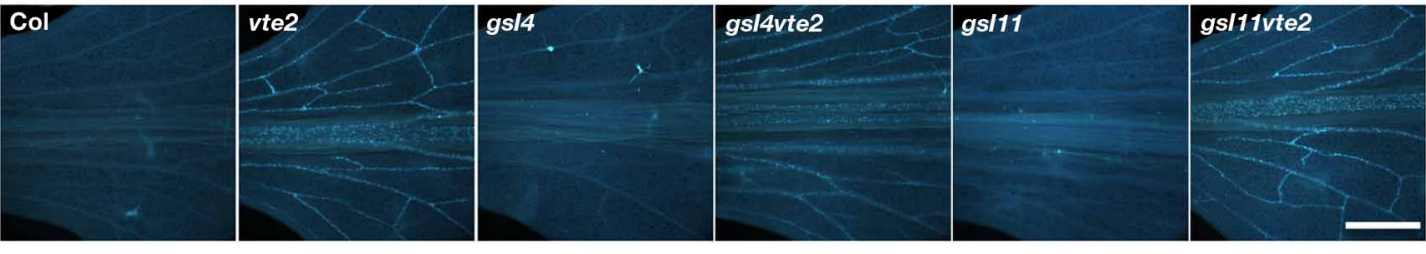

FIGURE 4 | Whole plant and vascular callose phenotypes of Col, vte2, gs/4, gs/4 vte2, gs/11, and gs/11 vte2. All genotypes were grown under permissive conditions for 4 weeks and then transferred to LT conditions for the specified periods previously shown to maximize each phenotype (Maeda et al., 2006). (A) Whole plant phenotype of the indicated genotypes before (top) and after (bottom) 28 days of LT treatment. Bar $=$ $2 \mathrm{~cm}$. (B) Aniline-blue positive fluorescence in the lower portions of leaves after 3 days of LT treatment. Samples for callose staining were fixed in the middle of the light cycle. Representative images are shown $(n=3)$. Bar $=1 \mathrm{~mm}$ callose was sometimes present but mostly rare to absent in all cell types of $\mathrm{Col}$ and gsl5 (Figures 6E,F). These results indicate that although GSL5 is responsible for the bulk of detectable callose deposition in LT treated vte2 (Figure 5A), callose synthase(s) other than GSL5 initiate the LT-induced callose deposition in transfer cells of vte 2 that may associate with the inhibition of photoassimilate export capacity in vte2.

\section{DISCUSSION}

Genome wide transcriptional responses to tocopherol deficiency have been extensively studied in animals using $\alpha$-tocopherol transfer protein knock-out mice (Ttpa-/-) (Gohil et al., 2003; Vasu et al., 2007, 2009) or animals fed with tocopherol-deficient diets (Barella et al., 2004; Rota et al., 2004, 2005; Nell et al., 2007; Oommen et al., 2007). In addition to oxidative stress related transcripts (Gohil et al., 2003; Jervis and Robaire, 2004; Hyland et al., 2006), tocopherols were found to modulate the expression of genes involved in hormone metabolism and apoptosis in the brain (Rota et al., 2005), lipid metabolism, inflammation, and immune system in the heart (Vasu et al., 2007), cytoskeleton modulation in lungs (Oommen et al., 2007), synaptic vesicular trafficking in liver (Nell et al., 2007), and muscle contractility and protein degradation in muscle (Vasu et al., 2009). In contrast to the broad effects of tocopherol deficiency on the animal transcriptome, we found that the absence of tocopherols in Arabidopsis leads to no significant changes in the global transcript profile of mature plants under permissive conditions. This finding extends a previous observation that Arabidopsis tocopherol-deficient mutants and wild type are virtually indistinguishable once they pass the oxidative stress bottlenecks of seed development and seedling germination (Maeda et al., 2006).

We previously showed that germinating seedlings of tocopherol-deficient Arabidopsis vte2 mutants exhibit massive levels of non-enzymatic lipid peroxidation (Sattler et al., 2004) resulting in differential expression of more than 700 genes when compared to wild type (Sattler et al., 2006). In contrast to these drastic biochemical and transcriptional changes in vte 2 seedlings, lipid peroxidation was not detectable in mature vte 2 leaves subject to LT treatment (Maeda et al., 2006, 2008) with only 77 genes having significantly altered expression (Tables 1, 2). Just 12 of these 77 genes are in common with the $>700$ altered genes in vte 2 seedlings, demonstrating that tocopherols play fundamentally distinct roles in seedlings and fully-expanded mature leaves. Moreover, of the 49 genes significantly upregulated in LT vte2 (Table 1), very few were also induced in response to other abiotic stresses (see Results). Instead approximately half of the 49 induced genes in LT vte2 were strongly and specifically upregulated by biotic and ozone treatments (Figure 3) with the latter significantly overlapping with transcriptional responses of plants to diseases (Eckeykaltenbach et al., 1994; Kangasjarvi 

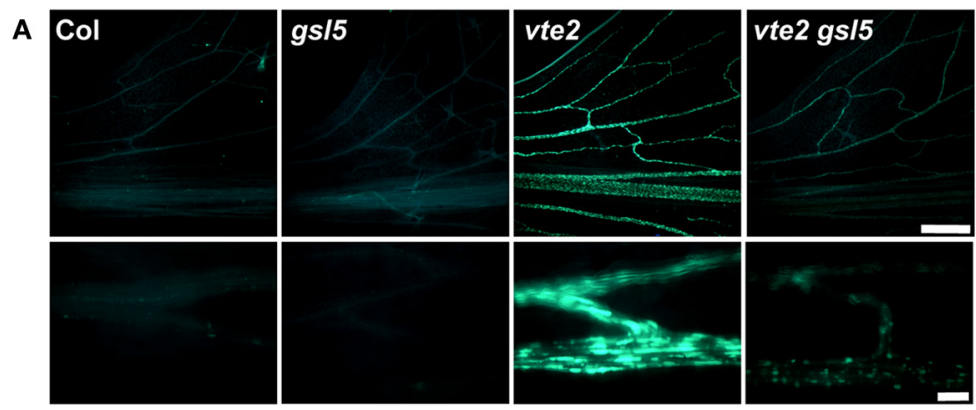

B Col
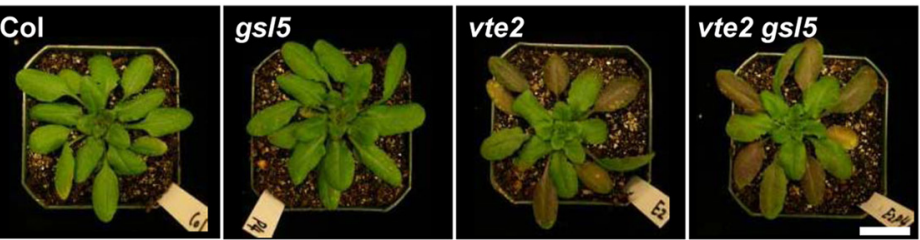

C

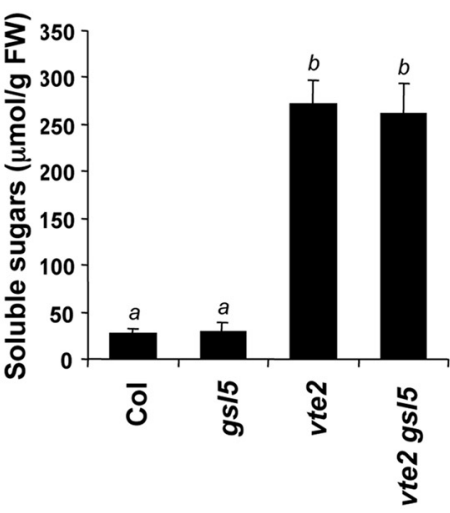

D

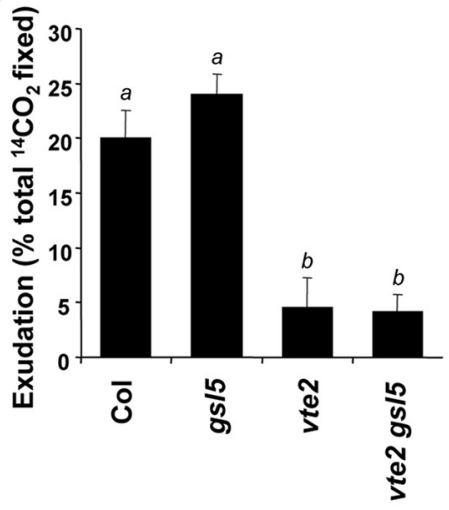

FIGURE 5 | Characterization of the gsI5 vte2 mutant at LT. Col, gs/5, vte2, and gs/5 vte2 were grown under permissive conditions for 4 weeks and transferred to LT conditions for the indicated times previously shown to maximize each phenotype (Maeda et al., 2006). (A) Aniline-blue positive fluorescence in the lower portions of leaves after 7 days of LT treatment. Samples for callose staining were fixed in the middle of the light cycle. Representative images are shown $(n=3)$. The bottom panels are higher magnification pictures of vasculature. Bars $=1 \mathrm{~mm}$ (top) and $100 \mu \mathrm{m}$ (bottom). (B) Whole plant phenotypes after 2 weeks of LT treatment. Bar $=$ $2 \mathrm{~cm}$. (C) Total soluble sugar content of mature leaves after 2 weeks of LT treatment. Data are means $\pm \mathrm{SD}(n=5)$. Non-significant groups are indicated by $a$ and $b(P<0.05)$. (D) ${ }^{14} \mathrm{C}$-labeled photoassimilate export capacity of mature leaves after one additional week of $\mathrm{LT}$ treatment. Data are means \pm $S D(n=5)$. Non-significant groups are indicated by $a$ and $b(P<0.05)$. et al., 1994; Ludwikow et al., 2004). Thus, in contrast to the strong oxidative response of the vte 2 seedling transcriptome to germination, the transcriptome of mature vte2 Arabidopsis leaves subjected to LT show a very limited oxidative stress response.

Prior studies have highlighted the involvement of tocopherols in extra-plastidic lipid metabolism under LT conditions (Maeda et al., 2006, 2008; Song et al., 2010). Based on these results, it might be expected that some genes related to lipid metabolism would be differentially expressed in vte 2 under LT. Surprisingly, however, only 2 of the 77 genes differentially expressed in LTtreated $v t e 2$ are involved in lipid metabolism. Both are lipase class 3 family proteins and proposed to have triacylglycerol lipase activities, with one (At2g30550) localized in the chloroplast and the other (At1g30370) to mitochondria. The majority of fatty acid desaturase genes in Arabidopsis, with the exception of FAD8 (Gibson et al., 1994), are not transcriptionally regulated in response to LT (Iba et al., 1993; Okuley et al., 1994;
Heppard et al., 1996) or alterations in the membrane fatty acid composition (Falcone et al., 1994). Thus, it seems that changes in extra-plastidic lipid metabolism in LT-treated vte2 plants are not transcriptionally-regulated but rather are regulated at the post-transcriptional level.

As with biochemical analysis of LT treated plants, experimental materials for the current microarray analysis were of necessity taken from whole leaves (see Materials and Methods) and it is possible that $v t e 2$ LT transcriptional "signatures" related to altered lipid metabolism or TCW synthesis are present but are restricted to such a small portion of specialized cell types (e.g., transfer cells) that their signals are diluted and difficult to identify in bulk leaf samples. Consistent with this idea, most of the genes identified as differentially expressed in LT-treated $v t e 2$ have low expression levels and attempts to verify their expression by traditional RNA gel blot analysis often failed (data not shown). It is possible that differential transcriptional responses may only be present in transfer 

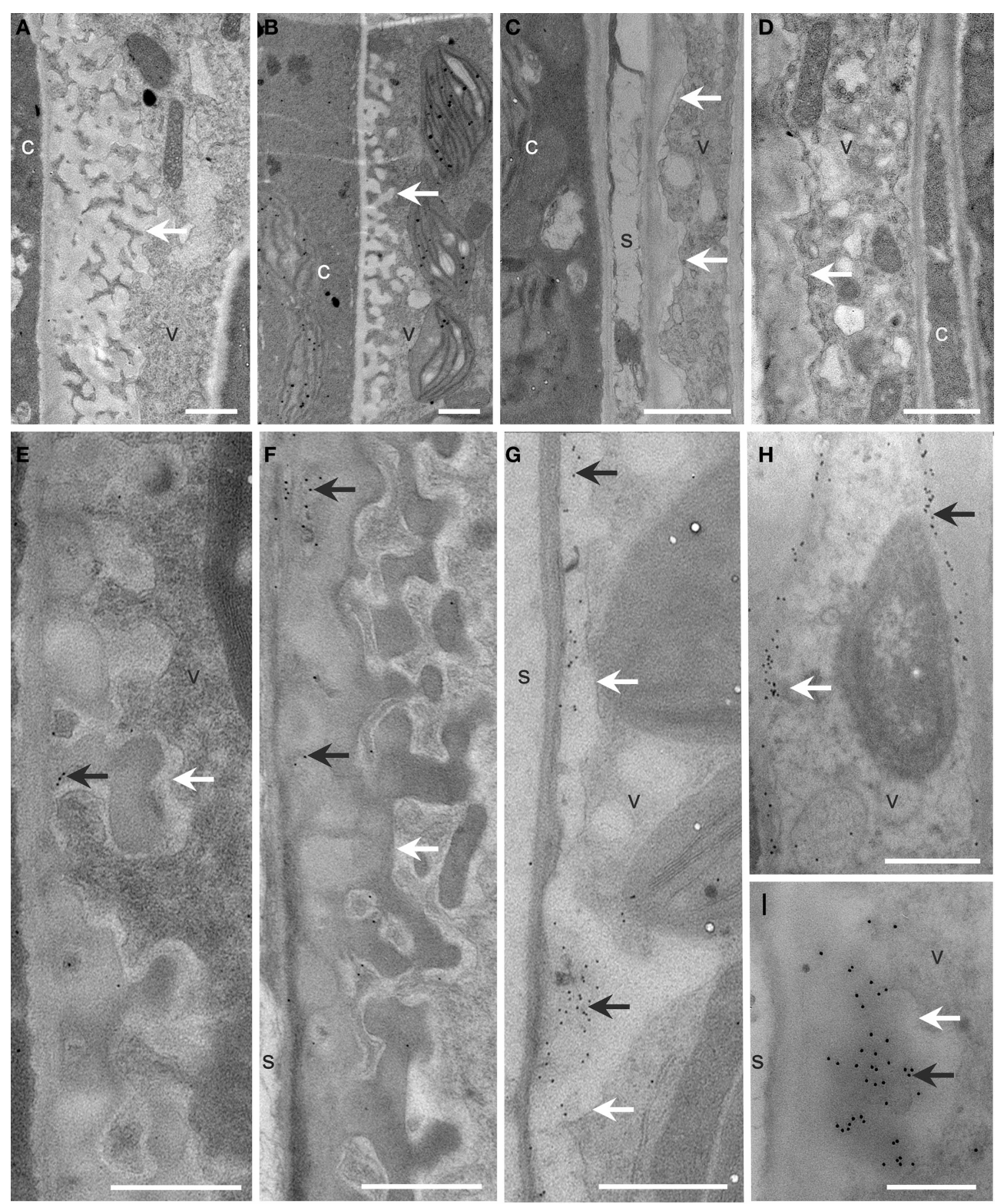

FIGURE 6 | Cellular structure and immunodetection of callose after 3 days of LT treatment. Col, gs/5, vte2, and gs/5 vte 2 were grown under permissive conditions for 4 weeks and transferred to LT conditions for 3 additional days. Col (A,E), gs/5 (B,F), vte2 (C, G), and gs/5 vte2 (D,H,I). Black arrows highlight wall ingrowths of phloem parenchyma transfer cells immunolabeled with anti- $\beta-1,3-$ glucan. White arrows mark transfer cell walls. C, companion cell; s, sieve element; $v$, vascular parenchyma transfer cell. Bars $=1 \mu \mathrm{m}(\mathbf{A}-\mathbf{H}), 0.5 \mu \mathrm{m}$ (I). cells of $v t e 2$, where endomembrane biogenesis is strongly induced (Maeda et al., 2006) and the deposition of callose and abnormal cell wall ingrowths occur (Figure 6). Future experiments utilizing in situ hybridization or laser-microdissection of vascular parenchyma cells would be necessary to directly test whether more than the 77 genes identified in this study show such cell specific expression at LT and whether additional genes are regulated by tocopherols and also may contribute to the LT-induced phenotype of $v t e 2$.

The biochemical phenotype of 48 h-LT-treated vte2 plants includes phloem transfer cell-specific callose deposition that spreads from the petiole to the upper part of the mature leaves and potentially impacts the capacity of source to sink photoassimilate transportation (Maeda et al., 2006). Consistent with these phenotypes, the expression of several genes (e.g., glycosyl transferases, glucanases) that are potentially involved in cell wall polymer modification in transfer cells (Dibley et al., 2009) were significantly upregulated in $v t e 2$ (Table 1 ). We also assessed the molecular nature of vasculature specific callose deposition observed in LT-treated vte2 and its impact on the photoassimilate export phenotype. Based on our microarray analysis GSL4 and GSL11 were more strongly upregulated in LT-treated vte2 
than in Col (Supplemental Figure S5) and therefore considered likely candidates for enzymes mediating the massive callose deposition observed in LT-treated vte2. However, introduction of gsl4 and gsl11 mutations into the vte 2 background did not visibly alter the callose deposition or overall phenotypes in LT-induced gsl4 vte 2 or gsl11 vte2 double mutants (Figure 4). Somewhat surprisingly, though GSL5 was not differentially expressed in LT-treated vte 2 and Col (Supplemental Figure S5), knocking out GSL5 in the vte 2 background eliminated the majority, but not all, of callose deposition in LT-treated vte2 (Figures 5A and 7), indicating that the bulk of vte2 callose deposition at LT is GSL5-dependent (Figure 7). Because GSL5 is also responsible for callose deposition in response to wounding (Jacobs et al., 2003), tocopherol deficiency may improperly stimulate the wound response pathway in transfer cells and lead to post-transcriptional activation of the GSL5 enzyme in transfer cells. However, Col and vte2 showed similar levels of wound-induced callose deposition (data not shown). Taken together, these data suggest that tocopherols are required for post-transcriptional activation of GSL5 in transfer cells by a mechanism that is likely independent of the wound-signaling pathway.

Unexpectedly, elimination of the majority of LT-inducible callose deposition by introduction of the gsl5 mutation into the $v t e 2$ background did not impact any of the other vte2 LT phenotypes (Figure 5). The vte2 mutant develops an abnormally thickened TCW structure with fewer to no reticulate wall ingrowths (Figure 6; Maeda et al., 2006, 2008). These structural anomalies are still retained in $v t e 2$ gsl5 (Figure 6). Thus, although it can

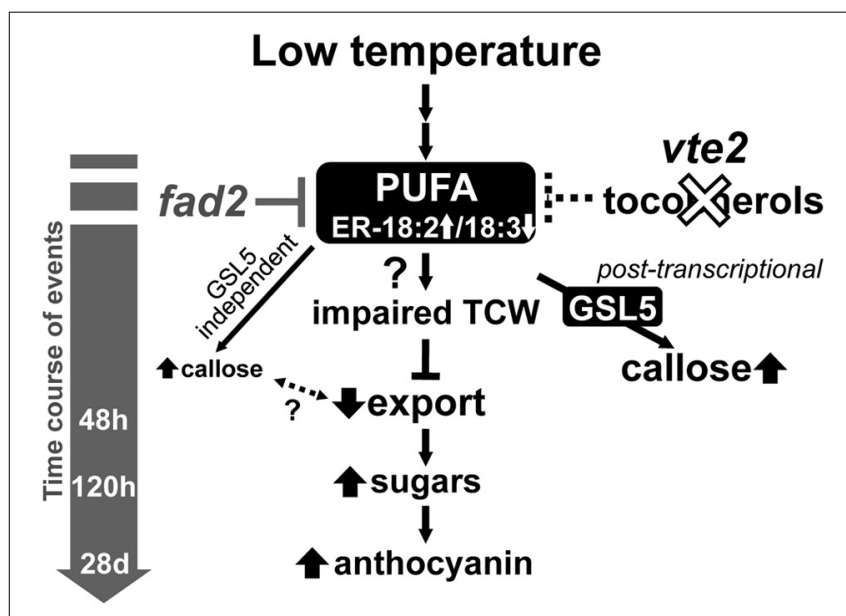

FIGURE 7 | A proposed model of the timing of biochemical changes in LT-induced phenotypes of tocopherol-deficient mutants. Tocopherol deficiency, e.g., by the vte 2 mutation, leads to constitutive alterations in the fatty acid composition of endoplasmic reticulum (ER) membrane lipids [i.e., reduced linolenic acid (18:3) and increased linoleic acid (18:2)]. These alterations are suppressed by the mutation of the ER FATTY ACID DESATURASE 2 gene (fad2), which also suppresses all of the LT-induced vte2 phenotypes (Maeda et al., 2008; Song et al., 2010). Subsequent vasculature-specific callose deposition is primarily mediated by the GSL5 enzyme and tocopherol-deficiency affects its activity post-transcriptionally. Although low levels of GSL5-independent callose deposition still occurs, loss of the massive GSL5-dependent callose deposition in transfer cells does not affected the subsequent defect in photoassimilate export in LT-treated vte2. be argued that the weaker, GSL5-independent fluorescent signals observed in the vasculature of vte2 gsl5 may still impact photosynthate transport and development of the full suite of vte2 LT phenotypes (Figures 6A, C,E,G, 7), these results indicate that the absolute level of callose deposition in transfer cells does not correlate with the photoassimilate export phenotype of vte2. This suggests that GSL5-dependent callose deposition is an event independent or downstream of the impaired photoassimilate export in LT-treated vte2 (Figure 7). Future studies will focus on whether GSL5-independent callose deposition is involved in the impaired photoassimilate transportation phenotype by introducing additional $g s l$ mutations into the vte2 gsl5 background (e.g., by constructing vte2 gsl5 gsl4 gsl11 quadruple mutant) to attempt elimination of all callose deposition in LT-treated vte2. Additional candidates to assess for this function include GSL8 and GSL12, which contribute to the control of symplastic trafficking through plasmodesmata (Guseman et al., 2010; Vaten et al., 2011).

Previous and current studies have demonstrated that tocopherols are required for normal development of TCW ingrowths in Arabidopsis leaves in response to LT (Figure 6; Maeda et al., 2006,2008 ). Although the precise underlying mechanism remains elusive, suppressor mutant analyses (Maeda et al., 2008; Song et al., 2010) and our recent transorganellar complementation study (Mehrshahi et al., 2013) suggest that deficiency in plastidlocalized tocopherols directly impacts ER membrane lipid biogenesis. Although speculative at this point, these alterations in ER membrane lipid metabolism may in turn impact other endomembrane-related processes, such as the massive increase in vesicular trafficking required for deposition of cell wall material in transfer cells at LT (Talbot et al., 2002; McCurdy et al., 2008). Further investigation of the molecular links between altered ER lipid metabolism and the impairment of TCW development in LT-treated vte2 (Figure 7) will illuminate the fundamental mechanisms underlying TCW development and function at LT.

\section{AUTHOR CONTRIBUTIONS}

Hiroshi Maeda, Wan Song, and Dean DellaPenna designed research; Hiroshi Maeda, Wan Song, and Tammy Sage performed research; Hiroshi Maeda, Wan Song, Tammy Sage, and Dean DellaPenna analyzed data; Hiroshi Maeda, Wan Song, and Dean DellaPenna wrote the paper.

\section{ACKNOWLEDGMENTS}

We are grateful to Maria Magallanes-Lundback for performing RNA extraction and labeling and the Research Technology Support Facility (RTSF) at Michigan State University for performing microarray analysis. We thank Kathy Sault for technical assistance with microscopy and other members of the DellaPenna lab for their critical advice and discussions. This work was supported by NSF grant MCB-023529 to Dean DellaPenna and a Connaught Award and NSERC of Canada Discovery Grant to Tammy Sage.

\section{SUPPLEMENTARY MATERIAL}

The Supplementary Material for this article can be found online at: http://www.frontiersin.org/journal/10.3389/fpls. 2014.00046/abstract 
Supplemental Figure S1 | RNA degradation plot for the 18 microarrays.

Supplemental Figure S2 | QC plot of 3': 5' ratios for control genes, percentage of present gene calls, and background levels of 18 microarrays.

Supplemental Figure S3 | Box plots of all perfect match (PM) intensities of non-normalized (upper) and quantile normalized (bottom) 18 array data set.

Supplemental Figure S4 | Whole plant phenotypes of the gs/5 vte2 mutant grown under permissive conditions for 4 weeks.

Supplemental Figure S5 | Gene expression profiles for the 12 GSL family members in 4-week old Col and vte2 treated at LT for 0,48 , and $120 \mathrm{~h}$.

\section{REFERENCES}

Barella, L., Muller, P. Y., Schlachter, M., Hunziker, W., Stocklin, E., Spitzer, V., et al. (2004). Identification of hepatic molecular mechanisms of action of alpha-tocopherol using global gene expression profile analysis in rats. Biochim. Biophys. Acta Mol. Basis Dis. 1689, 66-74. doi: 10.1016/j.bbadis.2004.02.002

Benjamini, Y., and Hochberg, Y. (1995). Controlling the false discovery rate - a practical and powerful approach to multiple testing. J. R. Stat. Soc. B Methodol. 57, 289-300.

Bolstad, B. M., Irizarry, R. A., Astrand, M., and Speed, T. P. (2003). A comparison of normalization methods for high density oligonucleotide array data based on variance and bias. Bioinformatics 19, 185-193. doi: 10.1093/bioinformatics/19.2.185

Bramley, P. M., Elmadfa, I., Kafatos, A., Kelly, F. J., Manios, Y., Roxborough, H. E., et al. (2000). Vitamin E. J. Sci. Food Agric. 80, 913-938. doi: 10.1002/(SICI) 10970010(20000515)80:7<913::AID-JSFA600>3.0.CO;2-3

Burton, G. W., and Ingold, K. U. (1981). Autoxidation of biological molecules. 1. The antioxidant activity of vitamin $\mathrm{E}$ and related chain-breaking phenolic antioxidants in vitro. J. Am. Chem. Soc. 103, 6472-6477. doi: 10.1021/ja00411a035

Collakova, E., and DellaPenna, D. (2001). Isolation and functional analysis of homogentisate phytyltransferase from Synechocystis sp. PCC 6803 and Arabidopsis. Plant Physiol. 127, 1113-1124. doi: 10.1104/pp.010421

Collakova, E., and DellaPenna, D. (2003). The role of homogentisate phytyltransferase and other tocopherol pathway enzymes in the regulation of tocopherol synthesis during abiotic stress. Plant Physiol. 133, 930-940. doi: 10.1104/pp.103.026138

DellaPenna, D., and Mene-Saffrane, L. (2011). "Vitamin E," in Advances in Botanical Research, eds F. Rebeille and R. Douces (Amsterdam: Elsevier Inc.), 179-227.

Dibley, S. J., Zhou, Y. C., Andriunas, F. A., Talbot, M. J., Offler, C. E., Patrick, J. W., et al. (2009). Early gene expression programs accompanying transdifferentiation of epidermal cells of Vicia faba cotyledons into transfer cells. New Phytol. 182, 863-877. doi: 10.1111/j.1469-8137.2009.02822.x

Eckeykaltenbach, H., Ernst, D., Heller, W., and Sandermann, H. (1994). Biochemical-plant responses to ozone. 4. Cross-induction of defensive pathways in parsley (Petroselinum crispum L.) plants. Plant Physiol. 104, 67-74. doi: 10.1104/pp.104.1.67

Erin, A. N., Spirin, M. M., Tabidze, L. V., and Kagan, V. E. (1984). Formation of alpha-tocopherol complexes with fatty acids - a hypothetical mechanism of stabilization of biomembranes by vitamin E. Biochim. Biophys. Acta 774, 96-102. doi: 10.1016/0005-2736(84)90279-7

Evans, H. M., and Bishop, K. S. (1922). On the existence of a hitherto unrecognized dietary factor essential for reproduction. Science 56, 650-651. doi: $10.1126 /$ science. 56.1458 .650

Fahrenholtz, S. R., Doleiden, F. H., Trozzolo, A. M., and Lamola, A. A. (1974). Quenching of singlet oxygen by alpha-tocopherol. Photochem. Photobiol. 20, 505-509. doi: 10.1111/j.1751-1097.1974.tb06610.x

Falcone, D. L., Gibson, S., Lemieux, B., and Somerville, C. (1994). Identification of a gene that complements an Arabidopsis mutant deficient in chloroplast omega-6 desaturase activity. Plant Physiol. 106, 1453-1459. doi: 10.1104/pp.106.4.1453

Gibson, S., Arondel, V., Iba, K., and Somerville, C. (1994). Cloning of a temperature-regulated gene encoding a chloroplast omega-3 desaturase from Arabidopsis thaliana. Plant Physiol. 106, 1615-1621. doi: 10.1104/pp.106. 4.1615
Gohil, K., Schock, B. C., Chakraborty, A. A., Terasawa, Y., Raber, J., Farese, R. V., et al. (2003). Gene expression profile of oxidant stress and neurodegeneration in transgenic mice deficient in alpha-tocopherol transfer protein. Free Radic. Biol. Med. 35, 1343-1354. doi: 10.1016/S0891-5849(03)00509-4

Guseman, J. M., Lee, J. S., Bogenschutz, N. L., Peterson, K. M., Virata, R. E., Xie, B., et al. (2010). Dysregulation of cell-to-cell connectivity and stomatal patterning by loss-of-function mutation in Arabidopsis chorus (glucan synthase-like 8). Development 137, 1731-1741. doi: 10.1242/dev.049197

Haritatos, E., Medville, R., and Turgeon, R. (2000). Minor vein structure and sugar transport in Arabidopsis thaliana. Planta 211, 105-111. doi: $10.1007 /$ s004250000268

Heppard, E. P., Kinney, A. J., Stecca, K. L., and Miao, G. H. (1996). Developmental and growth temperature regulation of two different microsomal omega-6 desaturase genes in soybeans. Plant Physiol. 110, 311-319. doi: 10.1104/pp. 110.1.311

Hofius, D., Hajirezaei, M. R., Geiger, M., Tschiersch, H., Melzer, M., and Sonnewald, U. (2004). RNAi-mediated tocopherol deficiency impairs photoassimilate export in transgenic potato plants. Plant Physiol. 135, 1256-1268. doi: 10.1104/pp.104.043927

Hohnjec, N., Lenz, F., Fehlberg, V., Vieweg, M. F., Baier, M. C., Hause, B., et al. (2009). The signal peptide of the Medicago truncatula modular nodulin MtNOD25 operates as an address label for the specific targeting of proteins to nitrogen-fixing symbiosomes. Mol. Plant Microbe Interact. 22, 63-72. doi: 10.1094/MPMI-22-1-0063

Hong, Z. L., Delauney, A. J., and Verma, D. P. S. (2001). A cell plate specific callose synthase and its interaction with phragmoplastin. Plant Cell 13, 755-768. doi: 10.1105/tpc.13.4.755

Hyland, S., Muller, D., Hayton, S., Stoecklin, E., and Barella, L. (2006). Cortical gene expression in the vitamin E-deficient rat: possible mechanisms for the electrophysiological abnormalities of visual and neural function. Ann. Nutr. Metab. 50, 433-441. doi: 10.1159/000094635

Iba, K., Gibson, S., Nishiuchi, T., Fuse, T., Nishimura, M., Arondel, V., et al. (1993). A gene encoding a chloroplast omega-3 fatty acid desaturase complements alterations in fatty acid desaturation and chloroplast copy number of the fad7 mutant of Arabidopsis thaliana. J. Biol. Chem. 268, 24099-24105.

Irizarry, R. A., Bolstad, B. M., Collin, F., Cope, L. M., Hobbs, B., and Speed, T. P. (2003). Summaries of affymetrix GeneChip probe level data. Nucleic Acids Res. 31:e15. doi: 10.1093/nar/gng015

Jacobs, A. K., Lipka, V., Burton, R. A., Panstruga, R., Strizhov, N., Schulze-Lefert, P., et al. (2003). An Arabidopsis callose synthase, GSL5, is required for wound and papillary callose formation. Plant Cell 15, 2503-2513. doi: 10.1105/tpc.016097

Jervis, K. M., and Robaire, B. (2004). The effects of long-term vitamin E treatment on gene expression and oxidative stress damage in the aging brown Norway rat epididymis. Biol. Reprod. 71, 1088-1095. doi: 10.1095/biolreprod.104.028886

Kagan, V. E. (1989). Tocopherol stabilizes membrane against phospholipase a, free fatty acids, and lysophospholipids. Ann. N.Y. Acad. Sci. 570, 121-135. doi: 10.1111/j.1749-6632.1989.tb14913.x

Kamal-Eldin, A., and Appelqvist, L. A. (1996). The chemistry and antioxidant properties of tocopherols and tocotrienols. Lipids 31, 671-701. doi: 10.1007/BF02522884

Kangasjarvi, J., Talvinen, J., Utriainen, M., and Katjalainen, R. (1994). Plant defence systems induced by ozone. Plant Cell Environ. 17, 783-794. doi: 10.1111/j.13653040.1994.tb00173.x

Liebler, D. C., and Burr, J. A. (1992). Oxidation of vitamin E during iron-catalyzed lipid-peroxidation - evidence for electron-transfer reactions of the tocopheroxyl radical. Biochemistry 31, 8278-8284. doi: 10.1021/bi00150a022

Ludwikow, A., Gallois, P., and Sadowski, J. (2004). Ozone-induced oxidative stress response in Arabidopsis: transcription profiling by microarray approach. Cell. Mol. Biol. Lett. 9, 829-842.

Maeda, H., Sage, T. L., Isaac, G., Welti, R., and DellaPenna, D. (2008). Tocopherols modulate extraplastidic polyunsaturated fatty acid metabolism in Arabidopsis at low temperature. Plant Cell 20, 452-470. doi: 10.1105/tpc.107. 054718

Maeda, H., Song, W., Sage, T. L., and DellaPenna, D. (2006). Tocopherols play a crucial role in low-temperature adaptation and phloem loading in Arabidopsis. Plant Cell 18, 2710-2732. doi: 10.1105/tpc.105.039404

McCurdy, D. W., Patrick, J. W., and Offler, C. (2008). Wall ingrowth formation in transfer cells: novel examples of localized wall deposition in plant cells. Curr. Opin. Plant Biol. 653-661. doi: 10.1016/j.pbi.2008.08.005 
Mehrshahi, P., Stefano, G., Andaloro, J. M., Brandizzi, F., Froehlich, J. E., and DellaPenna, D. (2013). Transorganellar complementation redefines the biochemical continuity of endoplasmic reticulum and chloroplasts. Proc. Natl. Acad. Sci. U.S.A. 110, 12126-12131. doi: 10.1073/pnas.1306331110

Mene-Saffrane, L., Jones, A. D., and DellaPenna, D. (2010). Plastochromanol-8 and tocopherols are essential lipid-soluble antioxidants during seed desiccation and quiescence in Arabidopsis. Proc. Natl. Acad. Sci. U.S.A. 107, 17815-17820. doi: 10.1073/pnas.1006971107

Nell, S., Bahtz, R., Bossecker, A., Kipp, A., Landes, N., Bumke-Vogt, C., et al. (2007). PCR-verified microarray analysis and functional in vitro studies indicate a role of alpha-tocopherol in vesicular transport. Free Radic. Res. 41, 930-942. doi: $10.1080 / 10715760701416988$

Nishimura, M. T., Stein, M., Hou, B. H., Vogel, J. P., Edwards, H., and Somerville, S. C. (2003). Loss of a callose synthase results in salicylic acid-dependent disease resistance. Science 301, 969-972. doi: 10.1126/science.1086716

Okuley, J., Lightner, J., Feldmann, K., Yadav, N., Lark, E., and Browse, J. (1994). Arabidopsis FAD2 gene encodes the enzyme that is essential for polyunsaturated lipid synthesis. Plant Cell 6, 147-158. doi: 10. 1105/tpc.6.1.147

Oommen, S., Vasu, V. T., Leonard, S. W., Traber, M. G., Cross, C. E., and Gohil, K. (2007). Genome wide responses of murine lungs to dietary alpha-tocopherol. Free Radic. Res. 41, 98-109. doi: 10.1080/10715760600935567

Provencher, L. M., Miao, L., Sinha, N., and Lucas, W. J. (2001). Sucrose export defectivel encodes a novel protein implicated in chloroplast-to-nucleus signaling. Plant Cell 13, 1127-1141. doi: 10.1105/tpc.13.5.1127

Richmond, T. A., and Somerville, C. R. (2000). The cellulose synthase superfamily. Plant Physiol. 124, 495-498. doi: 10.1104/pp.124.2.495

Rota, C., Barella, L., Minihane, A. M., Stocklin, E., and Rimbach, G. (2004). Dietary alpha-tocopherol affects differential gene expression in rat testes. IUBMB Life 56, 277-280. doi: 10.1080/15216540410001724133

Rota, C., Rimbach, G., Minihane, A. M., Stoecklin, E., and Barella, L. (2005). Dietary vitamin E modulates differential gene expression in the rat hippocampus: potential implications for its neuroprotective properties. Nutr. Neurosci. 8, 21-29. doi: 10.1080/10284150400027123

Russin, W. A., Evert, R. F., Vanderveer, P. J., Sharkey, T. D., and Briggs, S. P. (1996). Modification of a specific class of plasmodesmata and loss of sucrose export ability in the sucrose export defective1 maize mutant. Plant Cell 8, 645-658. doi: 10.1105/tpc.8.4.645

Sattler, S. E., Cahoon, E. B., Coughlan, S. J., and DellaPenna, D. (2003). Characterization of tocopherol cyclases from higher plants and cyanobacteria. Evolutionary implications for tocopherol synthesis and function. Plant Physiol. 132, 2184-2195. doi: 10.1104/pp.103.024257

Sattler, S. E., Gilliland, L. U., Magallanes-Lundback, M., Pollard, M., and DellaPenna, D. (2004). Vitamin E is essential for seed longevity, and for preventing lipid peroxidation during germination. Plant Cell 16, 1419-1432. doi: 10.1105/tpc.021360

Sattler, S. E., Mene-Saffrane, L., Farmer, E. E., Krischke, M., Mueller, M. J., and DellaPenna, D. (2006). Nonenzymatic lipid peroxidation reprograms gene expression and activates defense markers in Arabidopsis tocopherol-deficient mutants. Plant Cell 18, 3706-3720. doi: 10.1105/tpc.106.044065

Savidge, B., Weiss, J. D., Wong, Y. H. H., Lassner, M. W., Mitsky, T. A., Shewmaker, C. K., et al. (2002). Isolation and characterization of homogentisate phytyltransferase genes from Synechocystis sp PCC 6803 and Arabidopsis. Plant Physiol. 129, 321-332. doi: 10.1104/pp.010747

Schneider, C. (2005). Chemistry and biology of vitamin E. Mol. Nutr. Food Res. 49, 7-30. doi: 10.1002/mnfr.200400049

Smyth, G. K. (2005). "Limma: linear models for microarray data," in Bioinformatics and Computational Biology Solutions Using $R$ and Bioconductor, eds V. C. R.
Gentlemen, S. Dudoit, R. Irizarry, and W. Huber (New York, NY: Springer), 397-420. doi: 10.1007/0-387-29362-0_23

Song, W., Maeda, H., and DellaPenna, D. (2010). Mutations of the ER to plastid lipid transporters TGD1, 2, 3 and 4 and the ER oleate desaturase FAD2 suppress the low temperature-induced phenotype of Arabidopsis tocopherol-deficient mutant vte2. Plant J. 62, 1004-1018. doi: 10.1111/j.1365-313X.2010.04212.x

Stillwell, W., Dallman, T., Dumaual, A. C., Crump, F. T., and Jenski, L. J. (1996) Cholesterol versus alpha-tocopherol: effects on properties of bilayers made from heteroacid phosphatidylcholines. Biochemistry 35, 13353-13362. doi: 10.1021/bi961058m

Talbot, M. J., Offler, C. E., and McCurdy, D. W. (2002). Transfer cell wall architecture: a contribution towards understanding localized wall deposition. Protoplasma 219, 197-209. doi: 10.1007/s007090200021

Tappel, A. L. (1972). Vitamin E and free radical peroxidation of lipids. Ann. N.Y. Acad. Sci. 203, 12-28. doi: 10.1111/j.1749-6632.1972.tb27851.x

Vandewiel, C., Norris, J. H., Bochenek, B., Dickstein, R., Bisseling, T., and Hirsch, A. M. (1990). Nodulin gene expression and Enod2 Localization in effective, nitrogen-fixing and ineffective, bacteria-free nodules of alfalfa. Plant Cell 2, 1009-1017. doi: 10.1105/tpc.2.10.1009

Vasu, V. T., Hobson, B., Gohil, K., and Cross, C. E. (2007). Genome-wide screening of alpha-tocopherol sensitive genes in heart tissue from alphatocopherol transfer protein null mice (ATTP(-/-)). FEBS Lett. 581, 1572-1578. doi: 10.1016/j.febslet.2007.03.017

Vasu, V. T., Ott, S., Hobson, B., Rashidi, V., Oommen, S., Cross, C. E., et al. (2009). Sarcolipin and ubiquitin carboxy-terminal hydrolase 1 mRNAs are overexpressed in skeletal muscles of alpha-tocopherol deficient mice. Free Radic. Res. 43, 106-116. doi: 10.1080/10715760802616676

Vaten, A., Dettmer, J., Wu, S., Stierhof, Y. D., Miyashima, S., Yadav, S. R., et al. (2011). Callose biosynthesis regulates symplastic trafficking during root development. Dev. Cell 21, 1144-1155. doi: 10.1016/j.devcel.2011.10.006

Wang, X. Y., and Quinn, P. J. (2000). The location and function of vitamin E in membranes (review). Mol. Membr. Biol. 17, 143-156. doi: $10.1080 / 09687680010000311$

Zavaliev, R., Ueki, S., Epel, B. L., and Citovsky, V. (2011). Biology of callose (beta-1,3-glucan) turnover at plasmodesmata. Protoplasma 248, 117-130. doi: 10.1007/s00709-010-0247-0

Zimmermann, P., Hirsch-Hoffmann, M., Hennig, L., and Gruissem, W. (2004). GENEVESTIGATOR. Arabidopsis microarray database and analysis toolbox. Plant Physiol. 136, 2621-2632. doi: 10.1104/pp.104.046367

Conflict of Interest Statement: The authors declare that the research was conducted in the absence of any commercial or financial relationships that could be construed as a potential conflict of interest.

Received: 02 December 2013; accepted: 29 January 2014; published online: 19 February 2014.

Citation: Maeda H, Song W, Sage T and DellaPenna D (2014) Role of callose synthases in transfer cell wall development in tocopherol deficient Arabidopsis mutants. Front. Plant Sci. 5:46. doi: 10.3389/fpls.2014.00046

This article was submitted to Plant Physiology, a section of the journal Frontiers in Plant Science.

Copyright (c) 2014 Maeda, Song, Sage and DellaPenna. This is an open-access article distributed under the terms of the Creative Commons Attribution License (CC BY). The use, distribution or reproduction in other forums is permitted, provided the original author(s) or licensor are credited and that the original publication in this journal is cited, in accordance with accepted academic practice. No use, distribution or reproduction is permitted which does not comply with these terms. 